\title{
Neoadjuvant chemotherapy among patients treated for nonmetastatic breast cancer in a population with a high HIV prevalence in Johannesburg, South Africa
}

This article was published in the following Dove Press journal:

Cancer Management and Research

Paul Ruff, ${ }^{1,2}$ Herbert

Cubasch, ${ }^{2,3}$ Maureen Joffe, ${ }^{2,4}$

Evan Rosenbaum, ${ }^{5}$ Nivashni

Murugan, ${ }^{2,3}$ Ming-Chih Tsai, ${ }^{2,3}$

Oluwatosin Ayeni, ${ }^{2}$ Katherine

D Crew, ${ }^{5-7}$ Judith S Jacobson, 6,7

Alfred I Neugut ${ }^{5-7}$

'Division of Medical Oncology, Department of Internal Medicine,

University of the Witwatersrand, Faculty

of Health Sciences, ${ }^{2}$ Noncommunicable

Diseases Research Division, Wits

Health Consortium, University of the

Witwatersrand, Faculty of Health

Sciences, ${ }^{3}$ Department of Surgery,

Chris Hani Baragwanath Academic

Hospital and University of the

Witwatersrand, Faculty of Health

Sciences, ${ }^{4}$ MRC Developmental Pathways of Health Research Unit, Department of Paediatrics, University of Witwatersrand, Faculty of Health Sciences, Johannesburg, South Africa; ${ }^{5}$ Department of Medicine, College of Physicians and Surgeons,

Columbia University, ${ }^{6}$ Herbert Irving

Comprehensive Cancer Center, Columbia

University, ${ }^{7}$ Department of Epidemiology,

Mailman School of Public Health,

Columbia University, New York, NY, USA

Correspondence: Alfred I Neugut Herbert Irving Comprehensive Cancer

Center, Columbia University, 722 W 168th

Street, Room 725, New York, NY 10032.

USA

Tel + I $21230594 \mid 4$

$\mathrm{Fax}+\mathrm{I} 2123059413$

Email ain I@columbia.edu

Paul Ruff

Department of Internal Medicine, University of the Witwatersrand, 7 York Road,

Parktown 2193, Johannesburg, South Africa Email pruff@iafrica.com
Background: Neoadjuvant (primary) chemotherapy (NACT) is the standard of care for locally advanced breast cancer. It also allows for the short-term assessment of chemotherapy response; a pathological complete responses correspond to improved long-term breast cancer outcomes. In sub-Saharan Africa, many patients are diagnosed with large nonresectable tumors. We examined NACT use in breast cancer patients who visited public hospitals in Johannesburg, South Africa. Methods: We assessed demographic characteristics, tumor stage and grade, hormone receptor status, and human immunodeficiency virus (HIV) status of female patients diagnosed with nonmetastatic invasive carcinoma of the breast at Chris Hani Baragwanath Academic Hospital between January 1, 2009, and December 31, 2011. The patients received neoadjuvant, adjuvant, or no chemotherapy. Trastuzumab was unavailable. We developed logistic regression models to analyze the factors associated with NACT receipt in these patients.

Results: Of 554 women with nonmetastatic breast cancer, the median age at diagnosis was 52 years (range: $28-88$ years). Only $5.8 \%$ of patients were diagnosed with stage I disease; $49.3 \%$ and $44.9 \%$ were diagnosed with stages II and III, respectively. Most patients had hormoneresponsive tumors: luminal A, 38.1\%; luminal $\mathrm{B}_{1}$ (human epidermal growth factor receptor 2 [HER2]-negative and high grade), 12.5\%, and luminal $\mathrm{B}_{2}$ (HER2-positive any grade), $11.6 \%$; $11.6 \%$ had a HER2-enriched tumor and $20.6 \%$ a triple-negative tumor. Eighty (14.4\%) patients were HIV-positive. In total, 195 patients (35.2\%) received NACT, 264 (47.7\%) patients received adjuvant chemotherapy, and 95 patients (17.1\%) received no chemotherapy, including $62(11.2 \%)$ patients who received only hormonal therapy. Of patients receiving NACT, 125 (64.1\%) were evaluable for clinical response. Eighty $(64.0 \%)$ patients had a clinically significant response; 19 (15.2\%) patients had a stable disease, and 26 (20.8\%) patients had a progressive disease. Multivariate analysis showed age $<40$ years and disease stage to be independently associated with the receipt of NACT.

Conclusion: Most women receiving NACT with available response data showed a clinical benefit. Stage III disease at diagnosis and age $<40$ years were predictors of neoadjuvant versus adjuvant chemotherapy treatment.

Keywords: breast cancer, chemotherapy, neoadjuvant, South Africa, HIV, LMICs

\section{Introduction}

The incidence of breast cancer appears to be on the rise; 1.67 million new cases were diagnosed worldwide in 2012. ${ }^{1,2}$ Low- and middle-income countries (LMICs) have lower incidence rates than high-income countries (HICs) but account for the majority 
of breast cancer deaths. ${ }^{3}$ Patients in sub-Saharan Africa (SSA) have poor breast cancer outcomes by the standards of HICs; ${ }^{4}$ an estimated $50 \%-80 \%$ of breast cancers are locally advanced or metastatic at the time of diagnosis. ${ }^{5,6}$

Women with breast cancer in Africa have been reported to have earlier age at presentation and a higher proportion of more aggressive subtypes than women of European descent. ${ }^{7}$ However, our Soweto, Johannesburg, and South African (SA) National Cancer Registry data revealed that, as in the USA, $>60 \%$ of SA women present with the less aggressive, hormone-responsive luminal A and B breast cancer subtypes ${ }^{8,9}$ Furthermore, Surveillance, Epidemiology, and End Results (SEER) age-specific incidence rates of Black and White women are similar to those of the Johannesburg population (Figure 1).

Neoadjuvant (primary) chemotherapy (NACT) has become the standard of care for locally advanced breast cancer in HICs, particularly for more aggressive breast cancer subtypes such as human epidermal growth factor receptor 2 (HER2)-enriched breast cancer and triple-negative breast cancer (TNBC). NACT may facilitate breast-conserving surgery (BCS) by shrinking tumor size and decreasing nodal involvement, reduce the risk of adverse events after surgery, and provide early information about tumor response to chemotherapy. ${ }^{10-16}$ An important end point of NACT is the induction of a pathological complete response (pCR) in resected tumors. ${ }^{11,17}$ Because $\mathrm{pCR}$ has been associated with

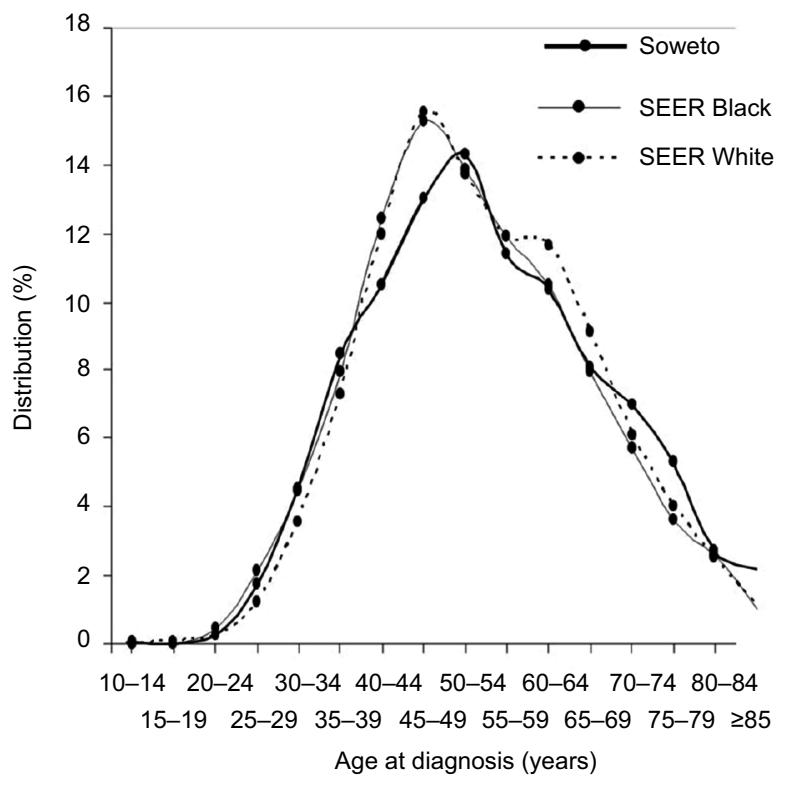

Figure I Age distribution of breast cancer incidence among women from Soweto, Johannesburg, and among US SEER Black and White women adjusted for Johannesburg population structure 2009-20I I.

Abbreviation: SEER, Surveillance, Epidemiology, and End Results. an increase in disease-free survival (DFS) and overall survival (OS), it is often used as a surrogate for those end points in research clinical trials of NACT. ${ }^{18-20}$

New therapeutic agents are increasingly being tested prior to surgery in the hope that they will show higher rates of pCR than older agents. ${ }^{21}$ Although many chemotherapeutic regimens have been used in the neoadjuvant setting, current guidelines recommend the use of an anthracycline-based regimen plus a taxane, along with HER2-targeted therapy when applicable and feasible. ${ }^{22}$

NACT is not well studied in breast cancer patients from LMICs, and the applicability or feasibility of applying HIC treatment guidelines to different patient populations is not known. ${ }^{23,24}$ Studies in SSA have small sample sizes, lack uniform standards for treatment, and use differing chemotherapeutic regimens. Overall response to NACT in SSA, measured clinically rather than pathologically, varies from $30 \%$ to $>90 \%$ in several reported studies. ${ }^{25-28}$

The Breast Health Global Initiative was convened to generate guidelines for breast cancer treatment in LMICs. The panel stratified care by the level of resource availability: basic, limited, enhanced, or maximal. The panel defined care at a basic level as what should be available in all health care systems, including the most under-resourced, and recommended anthracycline-based NACT as basic care for patients with locally advanced breast cancer. Treatment with taxanes and/or HER2-receptor inhibitors was recommended only for enhanced or maximal resource settings. ${ }^{5}$

Breast cancer care in much of SSA differs from the care provided to women in HICs because, in addition to resource constraints, LMICs have shortages of trained health care personnel, as well as cultural and educational barriers to care..$^{29,30}$

Laboratory and radiotherapy equipment and their maintenance are also suboptimal. For example, a survey of 19 medical facilities in 14 different African countries found that only 7 locations had well-maintained radiotherapy capabilities and 8 had the means to perform immunohistochemistry. ${ }^{31}$

Without proper cancer screening or community education to promote breast cancer awareness, diagnoses are made later in the course of the disease, compared with HICs. Tumor sizes, at diagnosis, $>10 \mathrm{~cm}$ accompanied by fungating breast masses are commonly reported. ${ }^{25,26,32}$ In HICs, where breast cancer awareness is widespread and screening is readily available and widely promoted, breast tumors are usually smaller at diagnosis than those in LMICs. ${ }^{33,34}$

Observations that women in SSA have lower rates of luminal A and higher rates of TNBC have led some investigators to believe that breast cancers in SSA are inherently more 
aggressive than those in HICs due to unique environmental exposures or genetic mutations. ${ }^{35}$ However, recent SA studies report breast cancer subtype distributions similar to those in the US populations. ${ }^{8,9}$

Compared with many of its neighbors, South Africa, an upper middle-income country, with an annual per capita gross domestic product (GDP) of $\sim$ US $\$ 7,000$ and purchasing power parity GDP of $\sim \$ 13,000,{ }^{36}$ has a relatively welldeveloped health care system, especially in the private sector. The public-sector health care service, which provides care to $\sim 85 \%$ of the population, includes a number of relatively wellequipped and staffed tertiary/quaternary hospitals, although in more rural settings, patients face barriers to care similar to those confronting patients in low-income countries. . $^{1,30,37}$

To the best of our knowledge, no data have been published on the use of NACT among SA breast cancer patients. We have therefore analyzed the factors associated with the use of NACT among patients diagnosed at the Surgical Breast Unit at Chris Hani Baragwanath Academic Hospital (CHBAH) and then treated at the Medical Oncology Unit at Charlotte Maxeke Johannesburg Academic Hospital (CMJAH), both of which are large academic hospitals in Johannesburg.

\section{Methods}

CHBAH is a tertiary-care public hospital located in Soweto, in the southwestern part of Johannesburg, Gauteng Province, South Africa. It serves as the referral facility for 3-4 million people who live within $60 \mathrm{~km}$ of the hospital, as well as patients from farther afield.

The public health system in South Africa has a hierarchical referral system: primary care doctors and nurses refer patients with more complex conditions to secondary-level hospitals, which refer patients requiring still more complex evaluation and care to tertiary/quaternary facilities. $\mathrm{CHBAH}$ has a specialized Surgical Breast Unit where 15-25 new patients per month are diagnosed with breast cancer. Breast diagnoses, surgery, and follow-up are performed at $\mathrm{CHBAH}$, while chemotherapy and radiation therapy are administered at CMJAH, $15 \mathrm{~km}$ from CHBAH.

Since 2006, the Surgical Breast Unit at CHBAH has maintained an electronic database of patient information. Since 2008, the Unit has been affiliated with the International Breast Centres Network and has standardized its treatment approach through weekly multidisciplinary meetings with cancer surgeons, medical oncologists, radiation oncologists, and palliative care specialists. In order to analyze the determinants of NACT, we reviewed the records of all female patients in the database diagnosed with Stages I-III invasive carcinoma of the breast from January 1, 2009, through December 31, 2011.

This study was approved by the University of the Witwatersrand Human Research Ethics Committee (Approval Number: M141129, dated December 5, 2014). The Committee did not ask us to obtain patients' consent because the study involved only retrospective medical record reviews. The data were anonymized for analysis to protect patients' confidentiality.

\section{NACT}

Most patients with Stages I and II breast cancer received primary surgery, while most patients with Stage III disease received NACT. The primary systemic regimens offered included 4 cycles of doxorubicin and cyclophosphamide followed by 4 cycles of paclitaxel $(\mathrm{AC} \rightarrow \mathrm{T})$ or 6 cycles of 5-fluorouracil, doxorubicin, and cyclophosphamide. HER2targeted therapy was not available for patients outside of a clinical trial. All patients with hormone-receptor-positive tumors received adjuvant tamoxifen. Patients with contraindications or intolerance of tamoxifen were offered anastrozole, an aromatase inhibitor, alone or, when applicable, in combination with goserelin, a long-acting gonadotropin-releasing hormone analog.

\section{Data and statistical analysis}

Data were abstracted from the electronic medical records in the CHBAH Surgical Breast Unit's database and the medical oncology records from CMJAH. The variables analyzed were patients' age, race, menopausal status, American Joint Committee on Cancer staging, histopathology diagnosis, tumor grade, estrogen receptor (ER)/progesterone receptor (PR) and HER2 status, as well as human immunodeficiency virus (HIV) status. Breast cancer subtypes were defined as luminal $\mathrm{A}(\mathrm{ER}+$ and/or PR+, HER2-, low/intermediate grade); luminal $\mathrm{B}_{1}(\mathrm{ER}+$ and/or $\mathrm{PR}+, \mathrm{HER} 2<3+$, high grade); luminal $\mathrm{B}_{2}(\mathrm{ER}+$ and/or PR+, HER2 3+, any grade); HER2enriched (ER-, PR-, HER2 3+, any grade), and TNBC (ER-, PR-, HER2-, any grade). Ki67 analysis was not performed during the period of the study, although it is now available. Immunohistochemistry HER2-equivocal $(2+)$ values were confirmed with in situ hybridization (ISH) results where they were available or defined as HER2- where ISH testing was not done.

We compared patients who received NACT to those who received adjuvant chemotherapy with respect to the variables listed above. Descriptive statistics such as frequencies and percentages were reported by means of tables for categorical 
variables using the Pearson's $\chi^{2}$ test and Fisher's exact test where appropriate (Table 1). Binary logistic regression was used to determine which factors were independently associated with NACT. All variables that were significant at $p<0.1$ on univariate analysis were evaluated in the multivariable model, and nonsignificant factors were dropped with stepwise backward regression. A two-sided $p$-value of $<0.05$ was considered significant throughout (Table 2 ). The analysis was

Table I Demographic and clinical characteristics of patients by chemotherapy category

\begin{tabular}{|c|c|c|c|c|c|c|c|c|c|}
\hline \multirow[t]{4}{*}{ Patient characteristics } & \multicolumn{9}{|c|}{ Chemotherapy category } \\
\hline & \multicolumn{2}{|c|}{ Neoadjuvant } & \multicolumn{2}{|c|}{ Adjuvant } & \multicolumn{2}{|c|}{ No chemotherapy } & \multicolumn{2}{|c|}{ Total } & \multirow[t]{3}{*}{$p$-value } \\
\hline & $\mathbf{n}$ & $\%$ & $\mathbf{n}$ & $\%$ & $\mathbf{n}$ & $\%$ & $\mathbf{n}$ & $\%$ & \\
\hline & 195 & 35.2 & 264 & 47.7 & 95 & 17.1 & 554 & 100 & \\
\hline Age group & & & & & & & & & $<0.001$ \\
\hline $18-39$ & 41 & 21.0 & 43 & 16.3 & 4 & 4.2 & 88 & 15.9 & \\
\hline $40-49$ & 48 & 24.6 & 70 & 26.5 & 5 & 5.3 & 123 & 22.2 & \\
\hline $50-59$ & 59 & 30.3 & 85 & 32.2 & 12 & 12.6 & 156 & 28.2 & \\
\hline $60-69$ & 29 & 14.9 & 47 & 17.8 & 15 & 15.8 & 91 & 16.4 & \\
\hline$\geq 70$ & 18 & 9.2 & 19 & 7.2 & 59 & 62.1 & 96 & 17.3 & \\
\hline Ethnicity & & & & & & & & & 0.984 \\
\hline Black & 179 & 91.8 & 241 & 91.3 & 86 & 90.5 & 506 & 91.3 & \\
\hline Asian & 3 & 1.5 & 7 & 2.7 & 3 & 3.2 & 13 & 2.3 & \\
\hline Mixed/colored & 6 & 3.1 & 8 & 3.0 & 3 & 3.2 & 17 & 3.1 & \\
\hline White & 7 & 3.6 & 8 & 3.0 & 3 & 3.2 & 18 & 3.2 & \\
\hline Clinical stage at diagnosis & & & & & & & & & $<0.001$ \\
\hline $\mathrm{IA}-\mathrm{B}$ & 0 & 0 & 21 & 8.0 & 11 & 11.6 & 32 & 5.8 & \\
\hline IIA-B & 17 & 8.7 & 204 & 77.3 & 52 & 54.7 & 273 & 49.3 & \\
\hline IIIA-C & 178 & 91.3 & 39 & 14.8 & 32 & 33.7 & 249 & 44.9 & \\
\hline Tumor grade & & & & & & & & & $<0.001$ \\
\hline I & 14 & 7.2 & 28 & 10.6 & 17 & 17.9 & 59 & 10.6 & \\
\hline 2 & 85 & 43.6 & 115 & 43.6 & 48 & 50.5 & 248 & 44.8 & \\
\hline 3 & 77 & 39.5 & 113 & 42.8 & 17 & 17.9 & 207 & 37.4 & \\
\hline Missing & 19 & 9.7 & 8 & 3.0 & 13 & 13.7 & 40 & 7.2 & \\
\hline Histology on diagnosis & & & & & & & & & 0.403 \\
\hline Invasive ductal carcinoma & 181 & 92.8 & 251 & 95.1 & 88 & 92.6 & 520 & 93.9 & \\
\hline Invasive lobular carcinoma & 6 & 3.1 & 9 & 3.4 & 6 & 6.3 & 21 & 3.8 & \\
\hline $\begin{array}{l}\text { Mixed ductal/lobular } \\
\text { carcinoma }\end{array}$ & 2 & 1.0 & 2 & 0.8 & 0 & 0 & 4 & 0.7 & \\
\hline Others & 4 & 2.1 & I & 0.4 & 0 & 0 & 5 & 0.9 & \\
\hline Missing & 2 & 1.0 & 1 & 0.4 & 1 & I.I & 4 & 0.7 & \\
\hline Molecular subtype & & & & & & & & & $<0.001$ \\
\hline Luminal A & 58 & 29.7 & 99 & 37.5 & 54 & 56.8 & 211 & 38.1 & \\
\hline Luminal $\mathrm{B}_{1}$ & 23 & 11.8 & 38 & 14.4 & 8 & 8.4 & 69 & 12.5 & \\
\hline Luminal $B_{2}$ & 29 & 14.9 & 25 & 9.5 & 10 & 10.5 & 64 & 11.6 & \\
\hline HER2-enriched & 20 & 10.3 & 37 & 14.0 & 7 & 7.4 & 64 & 11.6 & \\
\hline Triple-negative & 51 & 26.2 & 54 & 20.5 & 9 & 9.5 & 114 & 20.6 & \\
\hline No data & 14 & 7.2 & 11 & 4.2 & 7 & 7.4 & 32 & 5.8 & \\
\hline ER and PR expression & & & & & & & & & $<0.001$ \\
\hline ER+ alone & 28 & 14.4 & 29 & 11.0 & II & 11.6 & 68 & 12.3 & \\
\hline$E R+$ and $P R+$ & 77 & 39.5 & 124 & 47.0 & 65 & 68.4 & 266 & 48.0 & \\
\hline $\mathrm{PR}+$ alone & 7 & 3.6 & 15 & 5.7 & 2 & 2.1 & 24 & 4.3 & \\
\hline $\mathrm{ER}-$ and PR- & 83 & 42.6 & 96 & 36.4 & 17 & 17.9 & 196 & 35.4 & \\
\hline HER2 expression & & & & & & & & & 0.744 \\
\hline HER2-positive & 49 & 25.1 & 62 & 23.5 & 17 & 17.9 & 128 & 23.1 & \\
\hline HER2-negative & 149 & 74.9 & 202 & 76.5 & 78 & 82.1 & 426 & 76.9 & \\
\hline Total & 195 & 100 & 264 & 100 & 95 & 100 & 554 & 100 & \\
\hline HIV status on blood test & & & & & & & & & 0.008 \\
\hline Negative & 140 & 71.8 & 181 & 68.6 & 60 & 63.2 & 381 & 68.8 & \\
\hline Positive & 31 & 15.9 & 41 & 15.5 & 8 & 8.4 & 80 & 14.4 & \\
\hline Unknown & 24 & 12.3 & 42 & 15.9 & 27 & 28.4 & 93 & 16.8 & \\
\hline
\end{tabular}

Abbreviations: ER, estrogen receptor; HER2, human epidermal growth factor receptor 2; HIV, human immunodeficiency virus; PR, progesterone receptor. 
Table 2 Multivariable logistic regression model of predictors of neoadjuvant versus adjuvant chemotherapy

\begin{tabular}{llll}
\hline Predictors & OR & $\mathbf{9 5 \% ~ C l}$ & P-value \\
\hline HIV status & & & \\
Negative & 1.00 & Referent & \\
Positive & 0.73 & $0.3-1.8$ & 0.49 \\
Unknown & 1.06 & $0.4-2.6$ & 0.90 \\
Age group & & & \\
I8-39 & 1.00 & Referent & \\
$40-49$ & 0.32 & $0.1-0.9$ & 0.02 \\
$50-69$ & 0.37 & $0.2-0.9$ & 0.03 \\
$\geq 70$ & 0.18 & $0.05-0.7$ & 0.01 \\
Stage at diagnosis & & & \\
I \& II & 1.00 & Referent & \\
IIIA-C & 79.62 & $39.6-160.0$ & $<0.001$ \\
Molecular subtypes & & & \\
Luminal A & 1.00 & Referent & \\
Luminal B & 0.67 & $0.2-1.7$ & 0.40 \\
Luminal $B_{2}$ & 1.42 & $0.5-3.8$ & 0.49 \\
HER2-enriched & 0.48 & $0.2-1.2$ & 0.13 \\
TNBC & 1.50 & $0.7-3.4$ & 0.32 \\
\hline Abbreval
\end{tabular}

Abbreviations: HER2, human epidermal growth factor receptor 2; HIV, human immunodeficiency virus; TNBC, triple negative breast cancer.

performed by using Stata Version 14 (StataCorp LP, College Station, TX, USA).

\section{Results}

Of the 554 women who met the criteria for inclusion in our analysis, 506 (91.3\%) were Black and 211 (38.1\%) were aged $<50$ years, including $88(15.9 \%)$ women who were aged $<40$ years. At the time of diagnosis, 32 women $(6 \%)$ were found to have Stage I, 273 (49.1\%) Stage II, and 249 (44.9\%) Stage III disease. In line with our previous reports for SA populations, ${ }^{8,9} 211$ (38.1\%) patients had luminal A tumors; $69(12.5 \%)$ had luminal $\mathrm{B}_{1} ; 64(11.6 \%)$ had luminal $\mathrm{B}_{2}, 114$ (20.6\%) had HER2-enriched tumors, and 114 (20.6\%) had TNBC; and 32 (5.8\%) had missing receptor subtype data. Overall, 426 (76.9\%) patients had HER2- and 128 (23.1\%) had HER2+ tumors. Fifty-nine (10.6\%) patients had grade 1 tumors, 248 grade 2 (44.8\%), and 207 grade 3 (37.4\%) tumors, while 40 patients $(7.2 \%)$ had missing data for tumor grade. Of 461 patients whose HIV status was known, 80 (17.4\%) were HIV-positive (Table 1; 47 patients initially analyzed were found to have Stage IV disease and were excluded from the study).

Of the 459 patients $(82.9 \%)$ who received chemotherapy, 195 (42.5\%) received NACT, and 264 (57.5\%) received adjuvant chemotherapy. Among women who received NACT, 178 $(91.3 \%)$ had Stage III disease and 17 (8.7\%) Stage II disease. No women with Stage I disease were treated with NACT. In contrast, among women treated with adjuvant chemotherapy,
21 (8\%) had Stage I, 204 (77.3\%) Stage II, and 39 (14.7\%) Stage III disease.

Seven of the $195(3.6 \%)$ women who received NACT subsequently had BCS, while 69 (26.1\%) of the 264 women who had adjuvant chemotherapy had primary BCS. A further 15 women who had BCS were not referred for adjuvant chemotherapy.

Clinical response data were available for 125 (64.1\%) patients who received NACT, of whom $80(64.0 \%)$ patients were noted to have had a clinically significant response, 19 $(15.2 \%)$ to have stable disease, and $26(20.8 \%)$ to have the progression of their disease.

A multivariable logistic regression model controlling for age, HIV status, and tumor subtype found that women with Stage III breast cancers were $>79$ times as likely to receive NACT as women with Stage I or II breast cancer (Table 2).

Patients who tested positive for HIV and those with HER2-enriched tumors were less likely to receive NACT than uninfected patients and those with luminal A or B subtypes, but these differences were not statistically significant (Tables 1 and 2). Patients younger than 40 years were more likely to receive NACT than older patients, while race, tumor grade, and ER/PR status were not associated with the receipt of NACT (Table 2).

\section{Discussion}

In our sample of 554 women with nonmetastatic breast cancer, 17 of $273(6.2 \%)$ women with Stage II and 178 of 249 (71.5\%) women with Stage III disease received NACT. In our multivariable analysis, Stage III disease and age $<40$ years were the only independent predictors of receiving NACT (Table 2).

One of the purposes of NACT is to enable patients to receive more limited surgery than they would if the surgery preceded the chemotherapy. However, only seven of the 195 (3.6\%) NACT recipients in our sample actually had BCS, although 80 of 125 (64\%) of evaluable women receiving NACT had a clinically significant improvement, enabling mastectomy with clear surgical margins. As we have previously discussed, further study is needed to elucidate objective and pathological response rates and the extent to which NACT may facilitate BCS as opposed to mastectomy in our patient population. ${ }^{38}$

This study sheds light on the characteristics of female breast cancer treated in LMICs. About $35 \%$ of patients in this study received NACT, similar to that in HICs. Several large studies in the USA, including one of $>250,000$ patients, found that NACT was administered to $17 \%-36 \%$ of patients 
with nonmetastatic breast cancer. ${ }^{39-41}$ Similar to patients in our cohort, those in US samples were more likely to receive NACT if they had large tumors or late-stage disease. Most treatment guidelines call for NACT in patients with locally advanced breast cancer precisely because it can shrink inoperable tumors, facilitate definitive surgery, and increase the rates of breast conservation. ${ }^{14,22,42-46}$

Although not standard, preoperative chemotherapy may also be appropriate for patients with early-stage breast cancer. The National Comprehensive Cancer Network recommends NACT for operable tumors that have a high likelihood of responding to chemotherapy. NACT can provide predictive information on chemosensitivity, identify patients who may benefit from clinical trials of adjuvant therapies, and provide a setting to test novel medicines or biomarkers. ${ }^{22}$

In order for NACT to be predictive, the pathological response to chemotherapy must be ascertained. pCRs to NACT are strongly associated with improved DFS and OS among breast cancer patients. ${ }^{18,47}$ Biologic agents, such as trastuzumab, have been highly effective in enhancing pCR when used in combination with NACT; $>50 \%$ of patients achieve pCR in some studies.

\section{Strengths and limitations}

In our study, we did not have data on pathological response to NACT (given the limitations of retrospective studies of clinical record data), but 80 of 125 (64\%) of the women achieved a clinically significant tumor response. This response rate is comparable to, although slightly lower than, those seen previously in HICs, as well as other SSA countries. ${ }^{26,48,49}$ Future studies in this population should incorporate pathological response to NACT and determine whether NACT permits surgeons to perform more definitive surgery as well as BCS.

The proportion of women who tested positive for HIV in this study population (15.8\%) was not higher than the prevalence of HIV among women of similar age in South Africa ${ }^{50} \mathrm{HIV}$ has been implicated in the rise of some nonacquired immunodeficiency syndrome defining cancers, although not breast cancer. ${ }^{51-53}$ Prior studies of SA women with breast cancer and HIV have not found an association between HIV and tumor characteristics, such as stage, grade, or molecular subtypes. ${ }^{54}$ We did not find an association between NACT and HIV; HIV-positive patients (31 of 80 patients; $38.9 \%$ ) were just as likely as HIV-negative patients (140 of 381 patients; $36.7 \%$ ) to be referred for NACT (Table 2).

Findings among residents of Johannesburg may not be generalizable to more rural areas in South Africa or other countries in SSA. In addition, some data were missing regarding receptor status, lymphovascular invasion, HIV status, and pCR rates. Older women may have been underrepresented in our sample because of comorbid conditions and difficulties in accessing the health care system. ${ }^{37}$

The strengths of this study, however, include its relatively large sample size and the detailed data on tumor characteristics and breast cancer treatments.

\section{Conclusion}

NACT is the standard of care for women with locally advanced breast cancer in SA public hospitals. Most women who received NACT showed clinically significant benefit, although they generally did not receive biologic therapies such as trastuzumab. Only tumor stage and age $<40$ years were associated with the receipt of NACT. Future studies are needed to document the extent of clinical and pathological responses to NACT in South Africa, its acceptability to patients and their families, and its ability to improve breast cancer outcomes.

\section{Acknowledgments}

Drs Donald Dietz and Caroline Dickens provided significant assistance to the preparation of this manuscript for which we are grateful. We are also grateful to all the patients whose data were used for this study and to the medical and nursing teams at the Batho Pele Breast Unit of CHBAH and the Medical Oncology Unit at the CMJAH who have served and continue to serve their patients with skill and devotion. The research described in this report was supported by NCI 1R01CA192627 to Drs Jacobson, Joffe, Neugut, and Ruff; the South African Medical Research Council/University of the Witwatersrand Common Epithelial Cancer Research Centre led by Dr Ruff; and the Cancer Association of South Africa grant entitled "Down-staging and improving survival of breast cancer in South Africa" (Dr Cubasch).

\section{Author contributions}

All authors contributed toward data analysis, drafting and revising the paper and agree to be accountable for all aspects of the work.

\section{Disclosure}

Dr Ruff's institution received clinical research funding from Sanofi and Roche. Dr Neugut has served on the Medical Advisory Board of EHE, Intl. The other authors report no conflicts of interest in this work. 


\section{References}

1. Moodley J, Cairncross L, Naiker T, Momberg M. Understanding pathways to breast cancer diagnosis among women in the Western Cape Province, South Africa: a qualitative study. BMJ Open. 2016;6(1):e009905.

2. Ferlay J, Soerjomataram I, Dikshit R, et al. Cancer incidence and mortality worldwide: sources, methods and major patterns in GLOBOCAN 2012. Int J Cancer. 2015;136(5):E359-E386.

3. Bray F, Soerjomararam I. The changing global burden of cancer: Transitions in human development and implications for cancer prevention and control. In: Gelband H, Jha P, Sankaranarayanan R, Horton S, editors. Cancer: Disease Control Priorities, Third Edition (Volume 3). Washington (DC): The International Bank for Reconstruction and Development / The World Bank; 2015 Nov 1. Chapter 2, pp 23-44.

4. Sankaranarayanan R, Swaminathan R, Brenner H, et al. Cancer survival in Africa, Asia, and Central America: a population-based study. Lancet Oncol. 2010;11(2):165-173.

5. Eniu A, Carlson RW, Aziz Z, et al. Breast cancer in limited-resource countries: treatment and allocation of resources. Breast J. 2006;12 (Suppl 1): S38-S53.

6. Langa BC, Oliveira MM, Pereira SR, et al. Copy number analysis of the DLX4 and ERBB2 genes in South African breast cancer patients. Cytogenet Genome Res. 2015;146(3):195-203.

7. Eng A, McCormack V, dos-Santos-Silva I. Receptor-defined subtypes of breast cancer in indigenous populations in Africa: a systematic review and meta-analysis. PLoS Med. 2014;11(9):e1001720.

8. Dickens C, Duarte R, Zietsman A, et al. Racial comparison of receptordefined breast cancer in Southern African women: subtype prevalence and age-incidence analysis of nationwide cancer registry data. Cancer Epidemiol Biomarkers Prev. 2014;23(11):2311-2321.

9. McCormack VA, Joffe M, van den Berg E, et al. Breast cancer receptor status and stage at diagnosis in over 1,200 consecutive public hospital patients in Soweto, South Africa: a case series. Breast Cancer Res. 2013;15(5):R84.

10. von Minckwitz G, Blohmer JU, Raab G, et al. In vivo chemosensitivityadapted preoperative chemotherapy in patients with early-stage breast cancer: the GEPARTRIO pilot study. Ann Oncol. 2005;16(1):56-63.

11. von Minckwitz G, Costa SD, Raab G, et al. Dose-dense doxorubicin, docetaxel, and granulocyte colony-stimulating factor support with or without tamoxifen as preoperative therapy in patients with operable carcinoma of the breast: a randomized, controlled, open phase IIb study. J Clin Oncol. 2001;19(15):3506-3515.

12. Feldman LD, Hortobagyi GN, Buzdar AU, Ames FC, Blumenschein GR. Pathological assessment of response to induction chemotherapy in breast cancer. Cancer Res. 1986;46(5):2578-2581.

13. Kaufmann M, Hortobagyi GN, Goldhirsch A, et al. Recommendations from an international expert panel on the use of neoadjuvant (primary) systemic treatment of operable breast cancer: an update. J Clin Oncol. 2006;24(12):1940-1949.

14. Kaufmann M, von Minckwitz G, Mamounas EP, et al. Recommendations from an international consensus conference on the current status and future of neoadjuvant systemic therapy in primary breast cancer. Ann Surg Oncol. 2012;19(5):1508-1516.

15. Mauri D, Pavlidis N, Ioannidis JP. Neoadjuvant versus adjuvant systemic treatment in breast cancer: a meta-analysis. J Natl Cancer Inst. 2005;97(3):188-194.

16. Mieog JS, van der Hage JA, van de Velde CJ. Neoadjuvant chemotherapy for operable breast cancer. Br J Surg. 2007;94(10):1189-1200.

17. Fisher B, Brown A, Mamounas E, et al. Effect of preoperative chemotherapy on local-regional disease in women with operable breast cancer: findings from National Surgical Adjuvant Breast and Bowel Project B-18. J Clin Oncol. 1997;15(7):2483-2493.

18. von Minckwitz G, Untch M, Blohmer JU, et al. Definition and impact of pathologic complete response on prognosis after neoadjuvant chemotherapy in various intrinsic breast cancer subtypes. J Clin Oncol. 2012;30(15):1796-1804.
19. Rastogi P, Anderson SJ, Bear HD, et al. Preoperative chemotherapy: updates of National Surgical Adjuvant Breast and Bowel Project Protocols B-18 and B-27. J Clin Oncol. 2008;26(5):778-785.

20. Bear HD, Anderson S, Smith RE, et al. Sequential preoperative or postoperative docetaxel added to preoperative doxorubicin plus cyclophosphamide for operable breast cancer: National Surgical Adjuvant Breast and Bowel Project Protocol B-27. J Clin Oncol. 2006;24(13): 2019-2027.

21. Prowell TM, Pazdur R. Pathological complete response and accelerated drug approval in early breast cancer. $N$ Engl J Med. 2012;366(26): 2438-2441.

22. Breast Cancer (Version 2.2016). NCCN Guidelines Version 3.2017. Invasive Breast Cancer. November 10, 2017. https://www.nccn.org/ professionals/physician_gls/pdf/breast.pdf. Accessed 26 December, 2017.

23. Kantelhardt EJ, Cubasch H, Hanson C. Taking on breast cancer in East Africa: global challenges in breast cancer. Curr Opin Obstetr Gynecol. 2015;27(1):108-114.

24. Buccimazza I. Invited Commentary: the impact of neoadjuvant chemotherapy on patients with locally advanced breast cancer in a Nigerian semiurban teaching hospital: a single-center descriptive study. World $J$ Surg. 2010;34(8):1779-1781.

25. Arowolo OA, Akinkuolie AA, Lawal OO, Alatise OI, Salako AA, Adisa AO. The impact of neoadjuvant chemotherapy on patients with locally advanced breast cancer in a Nigerian semiurban teaching hospital: a single-center descriptive study. World J Surg. 2010;34(8):1771-1778.

26. Anyanwu SN, Nwose P, Ihekwoaba E, Mbaeri AT, Chukwuanukwu TO. Neoadjuvant chemotherapy for locally advanced premenopausal breast cancer in Nigerian women: early experience. Niger J Clin Pract. 2010;13(2):215-217.

27. Arowolo OA, Njiaju UO, Ogundiran TO, et al. Neo-adjuvant capecitabine chemotherapy in women with newly diagnosed locally advanced breast cancer in a resource-poor setting (Nigeria): efficacy and safety in a phase II feasibility study. Breast J. 2013;19(5): $470-477$.

28. Ogundiran TO, Ayandipo OO, Ademola AF, Adebamowo CA. Mastectomy for management of breast cancer in Ibadan, Nigeria. BMC Surg. 2013;13(1):59.

29. Francies FZ, Wainstein T, De Leeneer K, et al. BRCA1, BRCA2 and PALB2 mutations and CHEK2 c. 1100delC in different South African ethnic groups diagnosed with premenopausal and/or triple negative breast cancer. BMC Cancer. 2015;15(1):912.

30. Edge J, Buccimazza I, Cubasch H, Panieri E. The challenges of managing breast cancer in the developing world - a perspective from subSaharan Africa. S Afr Med J. 2014;104(5):377-379.

31. Williams CK, Stefan DC, Rawlinson F, Simbiri K, Mbulaiteye SM. The African Organisation for Research and Training in Cancer and its conferences: a historical perspective and highlights of the Ninth. Paper presented at: International Conference; November 21-24, 2013; Durban, South Africa.

32. Kingham TP, Alatise OI, Vanderpuye V, et al. Treatment of cancer in sub-Saharan Africa. Lancet Oncol. 2013;14(4):e158-e167.

33. Kopans DB. The effect of changes in tumor size on breast carcinoma survival in the U.S.: 1975-1999. Cancer. 2006;106(8):1863-1864.

34. Güth U, Huang DJ, Huber M, et al. Tumor size and detection in breast cancer: Self-examination and clinical breast examination are at their limit. Cancer Detect Prev. 2008;32(3):224-228.

35. Huo D, Ikpatt F, Khramtsov A, et al. Population differences in breast cancer: survey in indigenous African women reveals over-representation of triple-negative breast cancer. J Clin Oncol. 2009;27(27):4515-4521.

36. International Monetary Fund Annual Report 2016. Available from: https:// data.worldbank.org/indicator/NY.GDP.PCAP.PP.CD?locations=ZA. Accessed January 8, 2018.

37. Dickens C, Joffe M, Jacobson J, et al. Stage at breast cancer diagnosis and distance from diagnostic hospital in a periurban setting: a South African public hospital case series of over 1,000 women. Int J Cancer. 2014;135(9):2173-2182. 
38. Cubasch H, Joffe M, Ruff P, et al. Breast conservation surgery versus total mastectomy among women with localized breast cancer in Soweto, South Africa. PLoS One. 2017;12(8):e0182125.

39. Killelea BK, Yang VQ, Mougalian S, et al. Neoadjuvant chemotherapy for breast cancer increases the rate of breast conservation: results from the National Cancer Database. J Am Coll Surg. 2015;220(6): 1063-1069.

40. Mougalian SS, Soulos PR, Killelea BK, et al. Use of neoadjuvant chemotherapy for patients with stage I to III breast cancer in the United States. Cancer. 2015;121(15):2544-2552.

41. Mohiuddin JJ, Deal AM, Carey LA, et al. Neoadjuvant systemic therapy use for younger patients with breast cancer treated in different types of cancer centers across the United States. J Am Coll Surg. 2016;223(5):717-728.e714.

42. van der Hage JA, van de Velde CJ, Julien JP, Tubiana-Hulin M, Vandervelden C, Duchateau L. Preoperative chemotherapy in primary operable breast cancer: results from the European Organization for Research and Treatment of Cancer trial 10902. JClin Oncol. 2001;19(22): 4224-4237.

43. Cortazar P, Zhang L, Untch M, et al. Pathological complete response and long-term clinical benefit in breast cancer: the CTNeoBC pooled analysis. Lancet. 2014;384(9938):164-172.

44. Gralow JR, Burstein HJ, Wood W, et al. Preoperative therapy in invasive breast cancer: pathologic assessment and systemic therapy issues in operable disease. J Clin Oncol. 2008;26(5):814-819.

45. Wolmark N, Wang J, Mamounas E, Bryant J, Fisher B. Preoperative chemotherapy in patients with operable breast cancer: nine-year results from National Surgical Adjuvant Breast and Bowel Project B-18. J Natl Cancer Inst Monogr. 2001;(30):96-102.
46. Golshan M, Cirrincione CT, Sikov WM, et al. Impact of neoadjuvant chemotherapy in stage II-III triple negative breast cancer on eligibility for breast-conserving surgery and breast conservation rates: surgical results from CALGB 40603 (Alliance). Ann Surg. 2015;262(3):434 439; discussion 438-439.

47. Montagna E, Bagnardi V, Rotmensz N, et al. Pathological complete response after preoperative systemic therapy and outcome: relevance of clinical and biologic baseline features. Breast Cancer Res Treat. 2010;124(3):689-699.

48. Hortobagyi GN, Ames FC, Buzdar AU, et al. Management of stage III primary breast cancer with primary chemotherapy, surgery, and radiation therapy. Cancer. 1988;62(12):2507-2516.

49. Egwuonwu OA, Anyanwu SN, Nwofor AM. Efficacy of neoadjuvant chemotherapy in down staging locally advanced pre-menopausal breast cancer in Eastern Nigeria: is four courses adequate? J Cancer Res Ther. 2013;9(4):638-643.

50. Shisana O, Rehle T, Simbayi L, et al. South African National HIV Prevalence, Incidence And Behaviour Survey, 2012. Pretoria: Human Sciences Research Council; 2014

51. Shiels MS, Pfeiffer RM, Gail MH, et al. Cancer burden in the HIV-infected population in the United States. J Natl Cancer Inst. 2011;103(9):753-762.

52. Patel P, Hanson DL, Sullivan PS, et al. Incidence of types of cancer among HIV-infected persons compared with the general population in the United States, 1992-2003. Ann Intern Med. 2008;148(10):728-736.

53. Pantanowitz L, Dezube BJ. Evolving spectrum and incidence of nonAIDS-defining malignancies. Curr Opin HIV AIDS. 2009;4(1):27-34.

54. Cubasch H, Joffe M, Hanisch R, et al. Breast cancer characteristics and HIV among 1,092 women in Soweto, South Africa. Breast Cancer Res Treat. 2013;140(1):177-186.
Cancer Management and Research

\section{Publish your work in this journal}

Cancer Management and Research is an international, peer-reviewed open access journal focusing on cancer research and the optimal use of preventative and integrated treatment interventions to achieve improved outcomes, enhanced survival and quality of life for the cancer patient. The manuscript management system is completely online and includes

\section{Dovepress}

a very quick and fair peer-review system, which is all easy to use. Visit http://www.dovepress.com/testimonials.php to read real quotes from published authors. 